\title{
Probing Intrinsic Properties of Short Gamma-Ray Bursts with Gravitational Waves
}

\author{
Xilong Fan, ${ }^{1,2, *}$ Christopher Messenger, ${ }^{2}$ and Ik Siong Heng ${ }^{2}$ \\ ${ }^{1}$ School of Physics and Electronics Information, Hubei University of Education, 430205 Wuhan, China \\ ${ }^{2}$ SUPA, School of Physics and Astronomy, University of Glasgow, Glasgow G12 8QQ, United Kingdom
} (Received 13 July 2017; revised manuscript received 18 September 2017; published 3 November 2017)

Progenitors of short gamma-ray bursts are thought to be neutron stars coalescing with their companion black hole or neutron star, which are one of the main gravitational wave sources. We have devised a Bayesian framework for combining gamma-ray burst and gravitational wave information that allows us to probe short gamma-ray burst luminosities. We show that combined short gamma-ray burst and gravitational wave observations not only improve progenitor distance and inclination angle estimates, they also allow the isotropic luminosities of short gamma-ray bursts to be determined without the need for host galaxy or lightcurve information. We characterize our approach by simulating 1000 joint short gamma-ray burst and gravitational wave detections by Advanced LIGO and Advanced Virgo. We show that 90\% of the simulations have uncertainties on short gamma-ray burst isotropic luminosity estimates that are within a factor of two of the ideal scenario, where the distance is known exactly. Therefore, isotropic luminosities can be confidently determined for short gamma-ray bursts observed jointly with gravitational waves detected by Advanced LIGO and Advanced Virgo. Planned enhancements to Advanced LIGO will extend its range and likely produce several joint detections of short gamma-ray bursts and gravitational waves. Third-generation gravitational wave detectors will allow for isotropic luminosity estimates for the majority of the short gamma-ray burst population within a redshift of $z \sim 1$.

DOI: 10.1103/PhysRevLett.119.181102

Introduction.-The most likely candidate for the progenitor of a short duration gamma-ray burst (SGRB) event is the merger of a binary neutron star [1,2]. In this scenario such an event will be accompanied by the emission of a characteristic gravitational wave $(\mathrm{GW})$ signal detectable by the Advanced LIGO-Virgo GW interferometers as they approach design sensitivity [3]. A binary neutron star observation would follow the recent historic first detections of GWs from binary black hole mergers [4-6]. These merger events, while not consisting of neutron star components, were actively followed-up via multiple electromagnetic (EM) channels [7] including gamma-ray telescopes [8-10].

Because of the moderately well constrained properties of short-duration gamma-ray bursts (SGRBs), they are observed for only a narrow range of source orientations relative to the observers line of sight. Since GWs from compact binary coalescence (CBC) events are emitted broadly isotropically the expected rate of joint SGRB-GW detections is relatively low. This event rate for coincident SGRB-GW events has been discussed in [11-21].

Beyond rate estimates, there have also been a number of studies, including work presented in this Letter, exploring

Published by the American Physical Society under the terms of the Creative Commons Attribution 4.0 International license. Further distribution of this work must maintain attribution to the author(s) and the published article's title, journal citation, and DOI. how joint SGRB-GW detections can be used to enhance understanding of the underlying physical system. It has been shown by [22] that a three-dimensionally localized (sky direction and distance) SGRB in conjunction with the detection of the GW signal can improve the estimation of the inclination angle of the compact binary coalescence CBC. Also, although not truly a joint analysis, [23] discusses how a multidetector GW network alone can remove observational degeneracies in the inclination angle measurement allowing us to better understand off-axis SGRB events.

In this Letter, we focus on what can we learn from a single SGRB-GW detection. We model the system as being described by a set of parameters common to both the SGRB and GW with additional parameters associated with each phenomenon exclusively. Because of strong correlations between parameters, the combination of information from each observation channel allows improvement in common and exclusive parameters [24]. We specifically focus on the improved estimation of the GW inclination angle and the SGRB luminosity function in the likely scenario that there is a lack of an SGRB afterglow observation.

The statistical framework.-We use the framework set out in [24] to set up our analysis of joint EM-GW observations. Under this framework, for a given set of SGRB data, $\boldsymbol{S}$, and $\mathrm{GW}$ data, $\boldsymbol{D}$, we divide our observation parameters into three sets. The set of parameters common to both sets of observations is denoted by $\gamma$, which for joint SGRB-GW observations are Right Ascension $\alpha$, declination $\delta$, and distance $d$. The parameters that are distinct to 
either only the EM or GW observations are denoted by $\phi$ and $\omega$ respectively.

Our initial aim is to compute the posterior distribution on all parameters $\boldsymbol{\theta}=(\boldsymbol{\gamma}, \boldsymbol{\phi}, \boldsymbol{\omega})$ conditional on both data sets $\boldsymbol{S}, \boldsymbol{D}$ and any other implicit model assumptions denoted by I. We start by using Bayes' theorem to express the joint distribution on the complete parameters set as

$p(\boldsymbol{\theta} \mid \boldsymbol{S}, \boldsymbol{D}, I)=\frac{p(\boldsymbol{\gamma}, \boldsymbol{\omega}, \boldsymbol{\phi} \mid I) p(\boldsymbol{D} \mid \boldsymbol{\gamma}, \boldsymbol{\omega}, I) p(\boldsymbol{S} \mid \boldsymbol{\gamma}, \boldsymbol{\phi}, I)}{p(\boldsymbol{S}, \boldsymbol{D} \mid I)}$.

In [24], a model dependency was included that allowed the EM parameters to govern the probability that the GW and EM events originated from the same source. In this analysis, we ignore this complexity and assume that all SGRB events have been uniquely associated with a $\mathrm{GW}$ event.

The exclusive unknown SGRB parameters $\phi$ could contain many elements including the time of arrival of the SGRB in the detector frame, the duration of the burst, and spectral parameters for example. For simplicity, we include only the jet half-opening angle $\theta_{\text {jet }}$ and the isotropic SGRB luminosity $L_{\text {iso }}$. Similarly, we have choices regarding the EM data itself and in this analysis we assume that our relevant measurement information is contained within the measured peak flux $f_{\gamma}$. Hence, our EM likelihood becomes

$$
p(\boldsymbol{S} \mid \boldsymbol{\gamma}, \boldsymbol{\phi}, I)=\frac{1}{\sigma_{f_{\gamma}} \sqrt{2 \pi}} \exp \left(-\frac{\left(f_{\gamma}-f_{\mathrm{th}}\right)^{2}}{2 \sigma_{f_{\gamma}}^{2}}\right),
$$

where $f_{\text {th }}\left(d, L_{\text {iso }}, \theta_{\text {jet }}\right)$ is the expected value of the peak flux

$$
f_{\text {th }}\left(d, L_{\text {iso }}, \theta_{\text {jet }}\right)=\frac{L_{\text {iso }}}{4 \pi d^{2}} .
$$

The GW likelihood is obtained from the standard parameter estimation output of Bayesian Monte Carlo sampling algorithms. The input GW data consist of time series of strain data output from multiple detectors within a GW interferometer network. The parameter estimation information is available as a finite set of discrete samples drawn from the posterior $p(\gamma, \boldsymbol{\omega} \mid \boldsymbol{D}, I)$. We note that, in general, this posterior therefore already contains an assumed prior on the common and exclusive GW parameters that must be accounted for when converting back to the GW likelihood. The specific parameters included within the exclusive GW parameter set consist only of the neutron star masses $m_{1}$, $m_{2}$, the GW polarization angle $\psi$, and the binary inclination angle $l$. Note that the inclination angle is linked to the jet half-opening angle, since SGRBs are thought to be emitted perpendicular to the binary orbital plane (i.e., $l=0$ ). Though these two quantities are related, they describe different physical properties of the binary merger and are inferred from separate measurements. Thus, we do not list them as one of the common parameters.
We assume all parameter priors to be independent with the exception of the jet half-opening angle and the binary inclination angle. They can therefore be written as

$$
p(\boldsymbol{\theta})=p\left(m_{1} \mid I\right) p\left(m_{2} \mid I\right) p(\psi \mid I) p(d \mid I) p(L \mid I) p\left(\theta_{\text {jet }}, l \mid I\right) .
$$

The correlation between the $\theta_{\text {jet }}$ and $\iota$ priors is due to the fact that we assume that our sources have been jointly detected and therefore the jet half-opening angle must therefore be greater than the inclination angle. We also adopt the belief that the prior for the isotropic luminosity follows a power-law distribution [25] such that

$$
p\left(L_{\text {iso }} \mid I\right)=\frac{0.4}{L_{\min }}\left(\frac{L_{\text {iso }}}{L_{\min }}\right)^{-1.4}
$$

with a lower cutoff luminosity $L_{\min }=10^{49} \mathrm{erg} \mathrm{s}^{-1}$.

Simulation.-To characterize our method, we generated GW signals from binary neutron star (BNS) coalescences using LALAPPS_INSPINJ [26]. The signal parameters are drawn from the prior defined in Eq. (4) where masses are uniformly distributed on the range $(1.3,1.5) M_{\odot}$, the sky position is uniform on the sky, $\psi$ is uniform on the range $(0,2 \pi)$ and distance is selected uniformly in volume out to a maximum distance of $460 \mathrm{Mpc}$, equal to the horizon distance of the Advanced LIGO-Virgo network. At such distances, the effect of cosmological redshift is minimal and we do not include such effects in our simulations. The joint distribution on $\theta_{\text {jet }}$ and $\cos \imath$ is uniform under the constraints that $\cos \theta_{\text {jet }}<\cos \imath$ and $5^{\circ}<\theta_{\text {jet }}<30^{\circ}$ with values generated using rejection sampling. In total 1000 signals are generated and added to simulated noise from a three-detector network consisting of the two Advanced LIGO detectors (Hanford and Livingston) and Advanced Virgo at design sensitivity (see more details in [24]).

In order to construct an estimate of the GW likelihood, a kernel density estimation procedure [27] is used to compute the GW likelihood term $p(\boldsymbol{D} \mid \boldsymbol{\gamma}, \boldsymbol{\omega}, I)$ at any location in the $(\boldsymbol{\gamma}, \boldsymbol{\omega})$ parameter space. The discrete samples used as input are those generated from the posterior distribution on the GW parameters obtained using LALINFERENCE [28,29]. Since we require the likelihood, the prior distributions used in the generation of the posterior must be removed. Since the $\cos \imath$ prior is uniform, the likelihood and posterior are directly proportional and no change is necessary. The distance prior used to generate the posterior samples is uniform in volume, and hence $\propto d^{2}$. This is the same as that assumed for our general analysis as defined in Eq. (4) and so the $\mathrm{GW}$ posterior represents the combined terms $p(\boldsymbol{D} \mid \boldsymbol{\omega}, \boldsymbol{\gamma}, I) p(d \mid I)$.

The EM term $p(\boldsymbol{S} \mid \boldsymbol{\gamma}, \boldsymbol{\phi}, I)$ in the numerator of Eq. (1) is computed under the assumption that the SGRB skymap is both consistent with, and significantly more constraining than the GW skymap. This allows us to treat the sky 
position as known. As stated above, the jet half-opening angle is drawn jointly with the binary inclination angle consistent with the prior. The SGRB luminosity value is drawn from a power-law distribution according to Eq. (5). The measured flux $f_{\gamma}$ value is then drawn from a Gaussian distribution [consistent with our assumed likelihood, Eq. (2)] with mean $f_{\text {th }}$ given by Eq. (3) and a standard deviation equal to $30 \%$ of the mean.

The final joint posterior distribution on all signal parameters $p(\boldsymbol{\theta} \mid \boldsymbol{S}, \boldsymbol{D}, I)$ is obtained by computing the product of the estimated GW term and the analytic EM term multiplied by the priors on the all parameters according to Eq. (1). From this we can compute marginalized posterior distributions on any parameter, for example, the isotropic luminosity posterior for a joint SGRB-GW detection is given by

$$
p\left(L_{\text {iso }} \mid \boldsymbol{S}, \boldsymbol{D}, I\right)=\int d \boldsymbol{\theta}^{\left(\neq L_{\text {iso }}\right)} p(\boldsymbol{\theta} \mid \boldsymbol{S}, \boldsymbol{D}, I),
$$

where we have integrated over all parameters excluding the luminosity (indicated by the superscript $\neq L_{\text {iso }}$ on the $\boldsymbol{\theta}$ parameter set). Such a distribution represents the combined inference power of both the SGRB and GW observations.

Results.-The improvements in the accuracy of sky location and trigger time via a joint GW-SGRB observation have already been discussed in [30]. Here, we focus on the inference of distance, inclination angle and SGRB isotropic luminosity.

We select one of the simulations as a case study for illustrating the effects of the joint analysis (signal parameters given in Fig. 1). Contours representing the joint posterior on distance and $\cos l$ are plotted in Fig. 1 together with their marginalized distributions. The joint SGRB-GW analysis allows us to apply jet half-opening angle priors which constrain the inclination angle and consequently significantly improves the distance estimate. The combined SGRBGW posterior shown in Fig. 1 are produced by applying Eqs. (2)-(5) and are not obtained by the direct application of a threshold on the half opening angle posterior. For this particular case study, the $95 \%$ credible intervals for distance and $\cos l$ are improved by factors of $\sim 2.5$ and 8, respectively.

In Fig. 2 we show the marginalized probability density on the SGRB isotropic luminosity in our case study for two different scenarios. The first is the luminosity posterior assuming a joint SGRB-GW detection where we have marginalized all parameters excluding the luminosity using Eq. (6). The second curve is the posterior obtained using only an SGRB detection together with a correctly identified host galaxy at the true distance $d^{\prime}$. In this case the distance is then assumed known and the corresponding luminosity posterior is given by

$$
\begin{aligned}
& p\left(L_{\mathrm{iso}} \mid \boldsymbol{S}, d=d^{\prime}, I\right) \\
& \quad \propto \iint p(\boldsymbol{S} \mid \boldsymbol{\phi}, \boldsymbol{\gamma}, I) p(\boldsymbol{\phi}, \boldsymbol{\gamma} \mid I) \delta\left(d-d^{\prime}\right) d \phi d \gamma .
\end{aligned}
$$

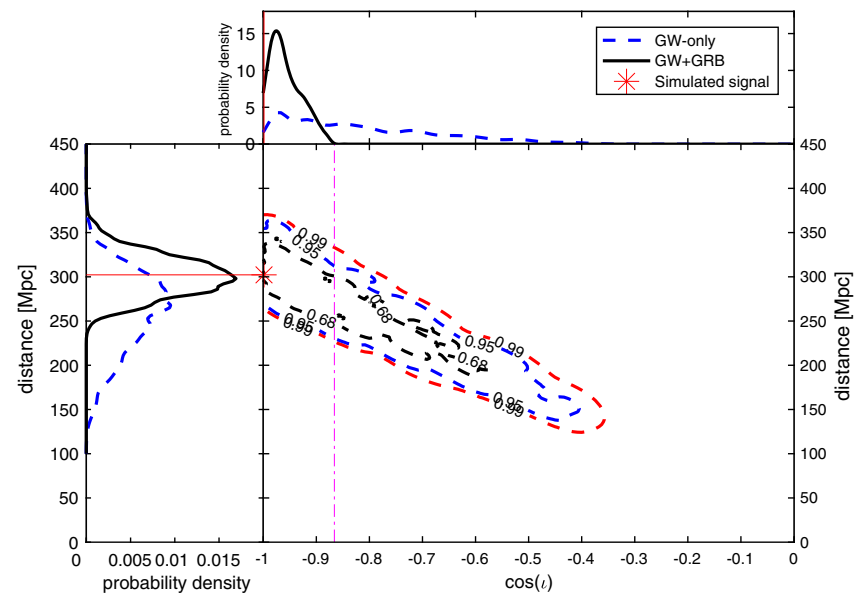

FIG. 1. Joint and marginalized posterior distributions on the distance and cosine inclination for a GW analysis and a joint SGRB-GW analysis. The example shown here is for a source at $d=302 \mathrm{Mpc}, \cos \imath=-0.99, \theta_{\text {jet }}=14.44^{\circ}, L_{\text {iso }}=5 \times 10^{49} \mathrm{erg}$. The distance and $\cos l$ values are indicated on the figure by an asterisk. The corresponding GW signal-to-noise ratio is 15.88 . Vertical lines in the marginalized posterior plots indicate the true simulated values. The restrictions imposed by the SGRB jet half-opening angle (vertical dash-dotted lines) cause significant reduction in the distance and inclination angle uncertainties.

In this case study the $95 \%$ credible intervals show that the joint SGRB-GW luminosity estimation is comparable with that of the SGRB-host galaxy observation.

Currently, isotropic luminosity estimates for SGRBs rely on obtaining redshift measurements of their assumed host galaxies. Only $\sim 30 \%$ of all detected SGRB have an identified host galaxy [31] ([32]), while all SGRBs observed in conjunction with GW counterparts will have a distance estimate directly from the GW observation. For SGRBs with identified host galaxies, the flux measurement uncertainty contributes to the spread in the isotropic luminosity posterior. For an SGRB-GW observation, all additional posterior width is due to the uncertainty remaining in the distance after the degeneracy between inclination angle and distance has been constrained by the joint SGRB-GW detection.

We examine the effectiveness of our proposed joint SGRB-GW analysis by examining the posterior credible intervals for the inferred source distance and SGRB isotropic luminosity using 1000 simulated signals as described previously.

In Fig. 3, we compare the 95\% credible intervals on the distance posterior distributions obtained using joint SGRBGW observations with those obtained for GW observations alone. Observing a SGRB in conjunction with a GW provides an additional constraints on the inclination angle which reduces the uncertainty in the source distance estimation. The $95 \%$ credible regions can be as much as $\sim 10$ times smaller for joint SGRB-GW analyses. In general, however, the median ratio is 0.65 . The fiducial detection threshold for ground based detectors is signal-to-noise ratio 


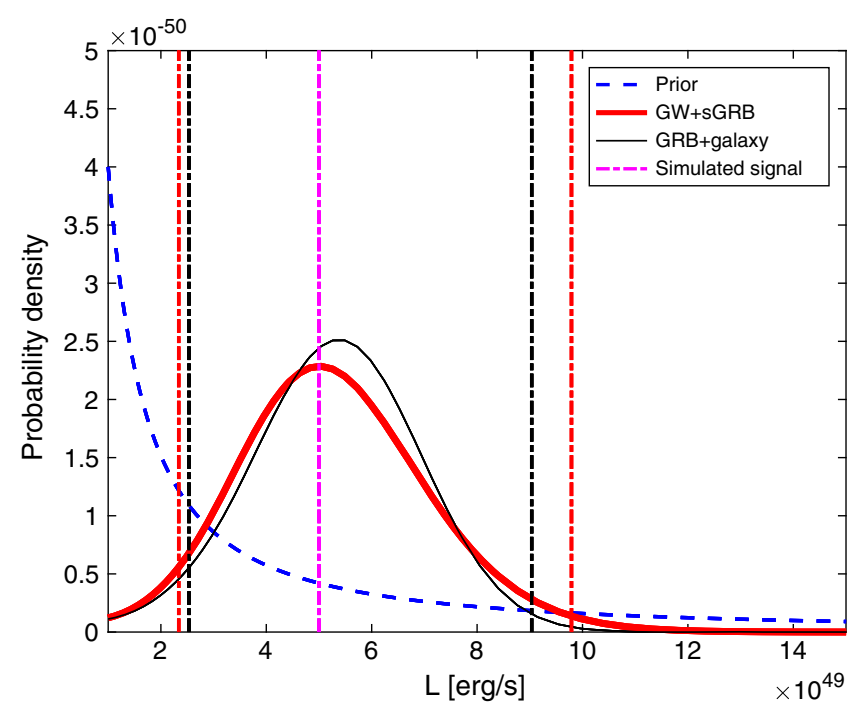

FIG. 2. The marginalized probability density on SGRB isotropic luminosity for our case study. The bold red curve shows the result for a joint SGRB-GW observation, and the thin blue curve shows the result for an SGRB observation with an identified host galaxy and corresponding exactly known distance. The vertical red and black dash-dotted lines represent the $95 \%$ credible regions for each case, respectively. The blue dashed curve shows the assumed prior for both cases [given by Eq. (5)]. The simulated value of isotropic luminosity is indicated by the vertical magenta dash-dotted line.

(SNR) 12 and if we restrict our analysis to only consider signals above this value then we find that the distance uncertainty reduction has a narrower range and shifts to lower values with a median ratio of 0.45 . The fact that it is most likely that a joint SGRB-GW observation will occur close to the horizon distance of the GW detector network is already accounted for in the distance prior. However, if we further restrict the analysis to detectable but distant signals such that the SNR $>12$ and the distances are $>300 \mathrm{Mpc}$ then the median ratio becomes 0.50 .

We also examine the ability of the joint SGRB-GW analysis to infer the SGRB isotropic luminosity and compare it to luminosities inferred using distances from identified SGRB host galaxies. We have assumed that all 1000 simulated signals have identified host galaxies and the host galaxy distances have been measured exactly. While this scenario is favorable for isotropic luminosity estimates obtained via host galaxy identification, we note that the host galaxy distance estimates can have a broad range of uncertainties (e.g. photometric redshifts would have greater uncertainty than spectroscopic redshifts, and host galaxies can be incorrectly identified) and can lead to considerable uncertainties in the corresponding luminosity estimate. However, to be conservative, we choose to compare scenarios that favor the SGRB host galaxy approach. With this in mind, in Fig. 3, we compare the 95\% credible intervals for the isotropic luminosity posterior distributions from the joint SGRB-GW analysis with that for source luminosities obtained via perfect galaxy host identification and corresponding error-free redshift measurements. We see that the credible intervals for the joint SGRB-GW analysis are typically within a factor of 2 of that obtained using the host galaxies. Given our optimistic assumptions regarding host galaxy identification, the relative level of uncertainty in the luminosity estimate achieved by the joint
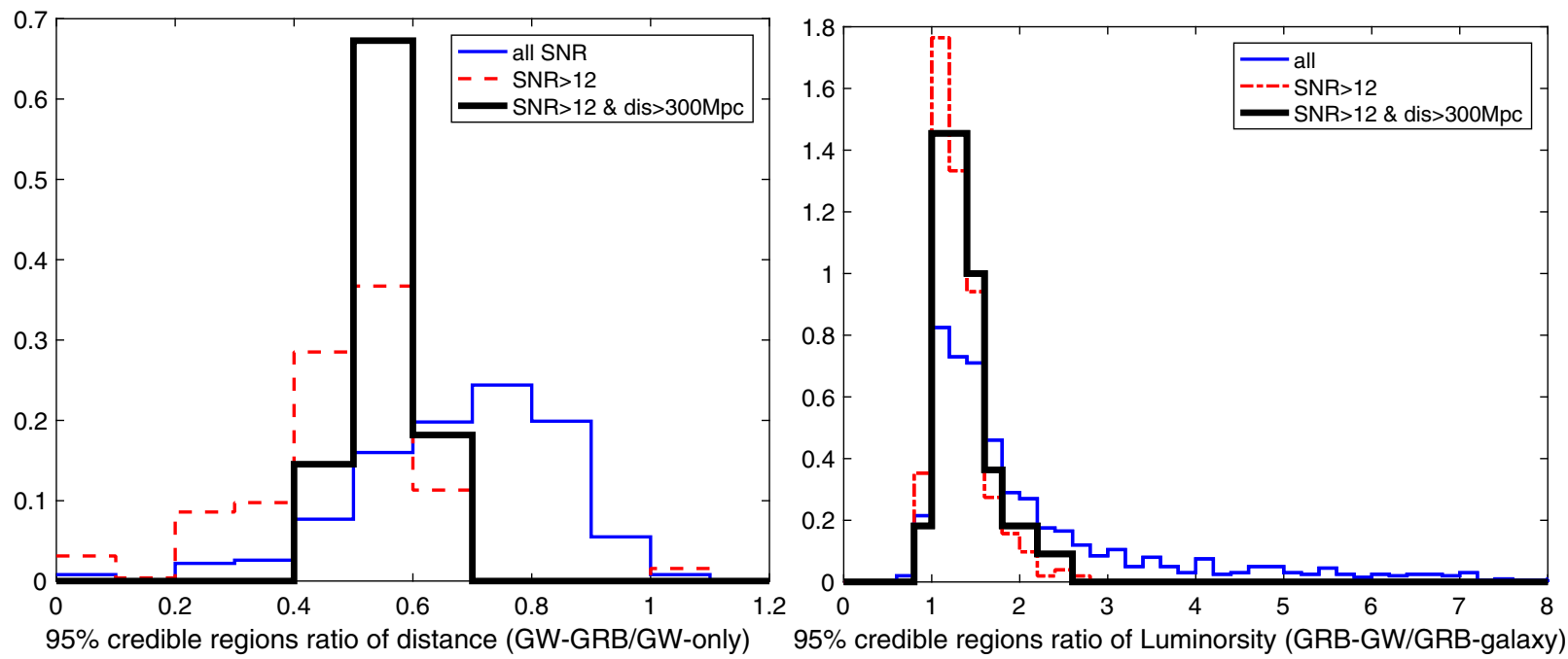

FIG. 3. (left) A histogram of the $95 \%$ credible interval ratios for distance. We take the ratios of the credible intervals between the joint SGRB-GW observations and the GW only scenarios. (right) A histogram of the $95 \%$ credible interval ratios for isotropic luminosity. We take the ratios of the credible intervals between the joint SGRB-GW observations and the SGRB-host galaxy scenarios. In both plots we consider three subsets of the results: all results (1000 samples, blue), SNR > 12 (256 samples, red) and SNR > 12 and distance $>300 \mathrm{Mpc}$ (55 samples, black). All cases are normalized according to their respective sample sizes. Note that the majority of the credible regions on isotropic luminosity obtained with the SGRB-GW analysis are similar to those obtained using host galaxy identification, with credible region ratios between $\sim 1$ and 2 . 
SGRB-GW analysis shows that we can obtain a reliable isotropic luminosity estimate for most SGRB-GW observations using our proposed method.

Discussion.-We have proposed a general procedure for the parameter estimation of two independent observations and applying it to reveal the nature of SGRBs using joint SGRB-GW observations. We have quantified the level to which the distance-inclination angle degeneracy inherent to GW observations can be broken with the inclusion of SGRB data. In addition to this, we have shown that through this joint analysis and the distance-inclination angle degeneracy breaking we are able to estimate the isotropic luminosity of the SGRB. The precision with which we are able to do this is comparable with the precision possible for SGRB analyses without GW counterparts but with a well-defined host galaxy.

A unique feature of our approach is that it will provide isotropic luminosity estimate for every joint SGRB-GW detection. This is in contrast to the fact that only 30\% of SGRB events have redshift estimates and hence an isotropic luminosity estimate. In the near term, the Advanced ground based GW detectors are likely to detect only a handful of joint events [17] and hence only make a small contribution to the $O(100)$ that will be known via SGRB observations on the same time scale. However, third generation $\mathrm{GW}$ detectors $[33,34]$ will be sensitive to BNS systems out to redshift $z \sim 1$ and will therefore jointly detect $\sim$ all SGRB events out to this distance. Joint detections will therefore provide $\sim$ twice as many luminosity measurements as are likely with SGRB events alone (assuming no improvement in redshift estimation).

By combining the information from EM and GW channels we have been able to quantify the improvements possible in the merger distance estimation. The spread in improvement ranges between a factor of 1-8 for all simulations and those detectable with SNR $>12$ showing improvements clustered around factors of $\sim 2$.

One possible extension to this work is to embed this analysis inside a hierarchical Bayesian model with the aim of inferring the SGRB isotropic luminosity distribution. In this work we have assumed a power-law prior distribution of the luminosity prior and the corresponding power-law index. A hierarchical procedure could be used to estimate this index and other parameters like the lower luminosity cutoff. It could also be used to perform model selection between, for example, single and broken power-law models. Additionally, nonparametric techniques such as Gaussian process modeling could provide estimates for the form of the isotropic luminosity distribution.

The method we have introduced in this Letter is not just applicable to joint observations using SGRBs and GWs. The power of the joint inference we describe can be applied to any multimessenger observations (two or more and not necessarily including GW observations) and will naturally exploit the parameter correlations between common parameters. As shown here, such correlations can lead to improved inference on other system parameters, and they could be applied to further astrophysical phenomena associated with GW events such as supernovae, kilonovae, or high energy neutrino observations.

We would like to acknowledge valuable input from $\mathrm{M}$. Hendry, F. Pannarale, D. Holz, and N. Tanvir. The authors also gratefully acknowledge the support of this research by the Royal Society, the Scottish Funding Council, the Scottish Universities Physics Alliance, and and the Science and Technology Facilities Council of the United Kingdom. X.F. acknowledges financial support from National Natural Science Foundation of China (Grants No. 11673008 and No. 11633001) and Newton International Fellowship Alumni Follow on Funding. C. M. and S. H. are supported by the Science and Technology Research Council (Grant No. ST/ L000946/1).

*Xilong.Fan@Glasgow.ac.uk

[1] D. Eichler, M. Livio, T. Piran, and D. N. Schramm, Nature (London) 340, 126 (1989).

[2] E. Berger, Annu. Rev. Astron. Astrophys. 52, 43 (2014).

[3] B. P. Abbott et al. (VIRGO, LIGO Collaborations), Living Rev. Relativity 19, 1 (2016).

[4] B. P. Abbott, R. Abbott, T. D. Abbott, M. R. Abernathy, F. Acernese, K. Ackley, C. Adams, T. Adams, P. Addesso, R. X. Adhikari et al., Phys. Rev. Lett. 116, 061102 (2016).

[5] B. P. Abbott, R. Abbott, T. D. Abbott, M. R. Abernathy, F. Acernese, K. Ackley, C. Adams, T. Adams, P. Addesso, R. X. Adhikari et al., Phys. Rev. X 6. 041015 (2016).

[6] B. P. Abbott, R. Abbott, T. D. Abbott, F. Acernese, K. Ackley, C. Adams, T. Adams, P. Addesso, R. X. Adhikari, V. B. Adya et al., Phys. Rev. Lett. 118, 221101 (2017).

[7] B. P. Abbott, R. Abbott, T. D. Abbott, M. R. Abernathy, F. Acernese, K. Ackley, C. Adams, T. Adams, P. Addesso, R. X. Adhikari et al., Astrophys. J. 826, L13 (2016).

[8] M. Ackermann, M. Ajello, A. Albert, B. Anderson, M. Arimoto et al., Astrophys. J. 823, L2 (2016).

[9] Z. Bagoly, D. Szécsi, L. G. Balázs, I. Csabai, I. Horváth, L. Dobos, J. Lichtenberger, and L. V. Tóth, Astron. Astrophys. 593, L10 (2016).

[10] V. Connaughton, E. Burns, A. Goldstein, L. Blackburn, M. S. Briggs, B.-B. Zhang, J. Camp, N. Christensen, C. M. Hui, P. Jenke et al., Astrophys. J. 826, L6 (2016).

[11] C. S. Kochanek and T. Piran, Astrophys. J. 417, L17 (1993).

[12] L. S. Finn, S. D. Mohanty, and J. D. Romano, Phys. Rev. D 60, 121101 (1999).

[13] H.-Y. Chen and D. E. Holz, Phys. Rev. Lett. 111, 181101 (2013).

[14] L. Z. Kelley, I. Mandel, and E. Ramirez-Ruiz, Phys. Rev. D 87, 123004 (2013).

[15] A. Dietz, N. Fotopoulos, L. Singer, and C. Cutler, Phys. Rev. D 87, 064033 (2013).

[16] D. M. Coward, M. Branchesi, E. J. Howell, P. D. Lasky, and M. Böer, Mon. Not. R. Astron. Soc. 445, 3575 (2014). 
[17] J. Clark, H. Evans, S. Fairhurst, I. W. Harry, E. Macdonald, D. Macleod, P. J. Sutton, and A. R. Williamson, Astrophys. J. 809, 53 (2015).

[18] Q. Chu, E. J. Howell, A. Rowlinson, H. Gao, B. Zhang, S. J. Tingay, M. Boër, and L. Wen, Mon. Not. R. Astron. Soc. 459, 121 (2016).

[19] X. Li, Y.-M. Hu, Y.-Z. Fan, and D.-M. Wei, Astrophys. J. 827, 75 (2016).

[20] B. Patricelli, M. Razzano, G. Cella, F. Fidecaro, E. Pian, M. Branchesi, and A. Stamerra, J. Cosmol. Astropart. Phys. 11 (2016) 056.

[21] H.-Y. Chen and D. E. Holz, Astrophys. J. 840, 88 (2017).

[22] N. Seto, Phys. Rev. D 75, 024016 (2007).

[23] K. G. Arun, H. Tagoshi, A. Pai, and C. K. Mishra, Phys. Rev. D 90, 024060 (2014).

[24] X. Fan, C. Messenger, and I. S. Heng, Astrophys. J. 795, 43 (2014).

[25] N. M. Lloyd-Ronning, X. Dai, and B. Zhang, Astrophys. J. 601, 371 (2004).

[26] https://www.lsc-group.phys.uwm.edu/daswg/projects/lalsuite .html.
[27] http://www.vicos.si/Research/ Multivariate_Online_Kernel_Density_Estimation.

[28] J. Aasi, J. Abadie, B. P. Abbott, R. Abbott, T. D. Abbott, M. Abernathy, T. Accadia, F. Acernese, C. Adams, T. Adams et al., Phys. Rev. D 88, 062001 (2013).

[29] J. Veitch and A. Vecchio, Phys. Rev. D 81, 062003 (2010).

[30] L. Blackburn, M. S. Briggs, J. Camp, N. Christensen, V. Connaughton, P. Jenke, R. A. Remillard, and J. Veitch, Astrophys. J. Suppl. Ser. 217, 8 (2015).

[31] This percentage is also consistent with counting entries with redshift estimates at https://swift.gsfc.nasa.gov/archive/ grb_table/.

[32] B. D. Metzger and E. Berger, Astrophys. J. 746, 48 (2012).

[33] B. Sathyaprakash, M. Abernathy, F. Acernese, P. Ajith, B. Allen, P. Amaro-Seoane, N. Andersson, S. Aoudia, K. Arun, P. Astone et al., Classical Quantum Gravity 29, 124013 (2012).

[34] B. P. Abbott et al., Classical Quantum Gravity 34, 044001 (2017). 